\title{
DECIDABILITY FOR SOME JUSTIFICATION LOGICS WITH NEGATIVE INTROSPECTION
}

\author{
THOMAS STUDER
}

\begin{abstract}
Justification logics are modal logics that include justifications for the agent's knowledge. So far, there are no decidability results available for justification logics with negative introspection. In this paper, we develop a novel model construction for such logics and show that justification logics with negative introspection are decidable for finite constant specifications.
\end{abstract}

§1. Introduction. Justification logic is a variant of modal logic that features explicit reasons for an agent's knowledge. Formally, justification logic includes statements of the form $t: F$ meaning $F$ is known for reason $t$ instead of the simple $\square F$ meaning $F$ is known. The evidence term $t$ in the statement $t: F$ can represent an informal justification why $F$ is known or a formal mathematical proof of $F$.

The first justification logic, the Logic of Proofs, was introduced by Artemov [1, $2]$ to provide the modal logic S4 with a provability semantics. Later it has been observed that justification logics also are a powerful tool in the context of epistemic logic, see for instance $[3,5,7,9]$.

From the beginning, decidability of justification logics has been an important issue. Already in [1], Artemov established decidability for the Logic of Proofs with any finite constant specification. The constant specification is an essential ingredient of a justification logic: it states which evidence constants justify which axioms of the logic. The concrete form of the constant specification matters a lot with respect to decidability. For instance, Kuznets [17] presented a decidable constant specification such that the corresponding Logic of Proofs is undecidable.

Mkrtychev [20] was able to show that the Logic of Proofs is decidable for schematic constant specifications. Kuznets [16] then provided decidability results for justification logics with schematic constant specifications that correspond to the modal logics K, KT, and K4. Vladimir Krupski [12, 13, 14, 15] obtained several decidability results for single conclusion justification logics. Decidability for justification logics that combine knowledge and justifications was established in $[6,24,25]$ where the constant specification is again required to be finite.

An excellent survey on these results as well as many new decidability theorems for justification logics can be found in Kuznets' PhD thesis [18]. He also 
presents a detailed analysis on what is needed to obtain decidability of a logic. In particular, he carefully states the following lemma, see [18, Lemma 4.3.1].

LEMMA 1. Let a finitely axiomatizable logic $\mathrm{L}$ be sound and complete with respect to a class of models $\mathcal{C}$, such that

1. the class $\mathcal{C}$ is recursively enumerable, and

2. the binary relation $\mathcal{M} \Vdash F$ between formulae and models from $\mathcal{C}$ is decidable.

Then $\mathrm{L}$ is decidable.

Artemov et al. [4] introduced the first justification logic with negative introspection. The current formulation, however, of justified negative introspection has been independently developed by Pacuit [21] and Rubtsova [23]. They prove several initial results for justification logics with negative introspection but what is missing so far are decidability results for those logics. In the present paper we establish first decidability theorems for justification logics with negative introspection. In particular, we show that the justification logics $\mathrm{J} 5 \mathrm{CS}, \mathrm{J} 45_{\mathrm{CS}}, \mathrm{JT} 5_{\mathrm{CS}}$, and $\mathrm{JT} 45_{\mathrm{CS}}$ are decidable for finite constant specifications CS.

One source of trouble for showing decidability for negative introspection is that the usual decidability proofs rely on minimal evidence relations. The evidence relation is that part of the semantics for justification logics that specifies which evidence terms are admissible evidence for which formulae. For logics without negative introspection, the evidence relation can be generated by a positive inductive definition and hence there is a minimal evidence relation. This does no longer work for negative introspection since negative introspection (as the name says) cannot be dealt with by a positive operator form.

Another problem one encounters is that justification logics with negative introspection are only sound with respect to strong models. A strong model requires that if, according to the model, a term $t$ is admissible evidence for a formula $F$, then $t: F$ is satisfied in the model. Justification logics without negative introspection are also sound with respect to models that do not fulfill this strong evidence property.

To solve the first problem, we develop a novel model construction that is based on non-monotone inductive definitions. Such inductive definitions are important for generalized recursion theory and the proof-theoretic analysis of strong systems, see for instance $[11,22]$. However, in the present paper we will only use a very weak form of non-monotone inductive definition and we will only be interested in models that are generated starting from a finite evidence basis. The second problem occurs in two places. First we have to guarantee that the inductive definition of our new model construction generates strong models. This is needed to show that the class of finitely generated strong models is recursively enumerable. Second we introduce a new form of generated submodel which preserves the property of being a strong model. This is needed to establish completeness with respect to finitely generated strong models.

The paper is organized as follows. In the next section we recall the definition of the justification logic JT45CS that corresponds to the traditional modal logic S5. We present a semantics (based on M-models [20]) for JT45 ${ }_{\text {CS }}$ and establish soundness and completeness with respect to arbitrary strong models. In order 
to show decidability of $\mathrm{JT} 45_{\mathrm{CS}}$, we need a class of models that satisfies the assumptions of Lemma 1. In Section 3 we introduce the class of finitely generated strong models and establish that

1. the satisfaction relation for finitely generated strong models is decidable, and

2. the class of finitely generated strong models is recursively enumerable.

Then in the following section we show that $\mathrm{JT} 45_{\mathrm{CS}}$ is complete with respect to finitely generated strong models. Hence decidability of JT45cs follows by Lemma 1. Section 5 discusses the situation for other logics. We show that the justification logics $\mathrm{J} 5_{\mathrm{CS}}, \mathrm{J} 45_{\mathrm{CS}}$, and $\mathrm{JT} 5_{\mathrm{CS}}$ are decidable, too. However, we also show that the condition of a finite constant specification is very important for our proof.

Finally, we conclude the paper by mentioning some open problems and future work.

Acknowledgments. We would like to thank Samuel Bucheli and Roman Kuznets for carefully proof reading this manuscript and for providing many valuable comments. We are also grateful to the anonymous referee who helped to improve the quality of this paper.

§2. Justification Logic. Justification terms are built from countably many constants $c_{i}$ and variables $x_{i}$ according to the following grammar:

$$
t:==c_{i}\left|x_{i}\right| t \cdot t|t+t| ! t \mid ? t \quad .
$$

We denote the set of terms by Tm. Formulae are built from countably many atomic propositions $p_{i}$ according to the following grammar:

$$
F:==p_{i}|\neg F| F \rightarrow F \mid t: F \text {. }
$$

Prop denotes the set of atomic propositions and Fm denotes the set of formulae. We use $\perp$ as an abbreviation for $p \wedge \neg p$ where $p$ is some fixed atomic proposition. For a formula $F$, we denote the set of subformulae of $F$ by $\operatorname{subfml}(F)$.

The set of axioms JT45 consists of:

1. all propositional tautologies

2. $t:(A \rightarrow B) \rightarrow(s: A \rightarrow t \cdot s: B)$

3. $s: A \rightarrow s+t: A$ and $t: A \rightarrow s+t: A$

Application

4. $t: A \rightarrow A$

5. $t: A \rightarrow ! t: t: A$

6. $\neg t: A \rightarrow ? t: \neg t: A$ Sum

Reflection Positive Introspection Negative Introspection

A constant specification CS for JT45 is any subset

$$
\mathrm{CS} \subseteq\{c: A \mid c \text { is a constant and } A \text { is an axiom of JT45 }\} .
$$

For a constant specification CS the deductive system JT45 CS is the Hilbert system given by the axioms above and by the rules modus ponens and axiom necessitation:

$$
\frac{A \quad A \rightarrow B}{B}(\mathrm{MP}), \quad \frac{c: A \in \mathrm{CS}}{c: A}(\mathrm{AN})
$$


We introduce a semantics for JT45 cs that is based on M-models [20]. A closely related semantics for justification logics with negative introspection has recently been studied in [19].

Definition 2 (Evidence relation). Let CS be a constant specification. An admissible evidence relation $\mathcal{E}$ is a subset of $\mathrm{Tm} \times \mathrm{Fm}$ that satisfies the following conditions:

1. if $c: A \in C S$, then $(c, A) \in \mathcal{E}$

2. if $(s, A) \in \mathcal{E}$ or $(t, A) \in \mathcal{E}$, then $(s+t, A) \in \mathcal{E}$

3. if $(s, A \rightarrow B) \in \mathcal{E}$ and $(t, A) \in \mathcal{E}$, then $(s \cdot t, B) \in \mathcal{E}$

4. if $(t, A) \in \mathcal{E}$, then $(! t, t: A) \in \mathcal{E}$

5. if $(t, A) \notin \mathcal{E}$, then $(? t, \neg t: A) \in \mathcal{E}$

Definition 3 (Model). Let CS be a constant specification. A model is a pair $\mathcal{M}=(\mathcal{E}, \nu)$ where

- $\mathcal{E}$ is an admissible evidence relation,

- $\nu \subseteq$ Prop is a valuation.

DEFinition 4 (Satisfaction relation). The satisfaction relation of formula $F$ in a model $\mathcal{M}=(\mathcal{E}, \nu)$ is defined by induction on the structure of $F$ where the cases for propositions and boolean connectives are as usual and $\mathcal{M} \Vdash t: A$ if and only if

1. $(t, A) \in \mathcal{E}$ and

2. $\mathcal{M} \Vdash A$

JT45 CS is only sound with respect to so-called strong models that are introduced next. Thus, the notion of validity will refer to strong models only.

Definition 5 (Strong model). A model $\mathcal{M}=(\mathcal{E}, \nu)$ is called strong model if it satisfies the strong evidence property: for all terms $t$ and all formulae $A$

$$
(t, A) \in \mathcal{E} \quad \Longrightarrow \quad \mathcal{M} \Vdash t: A \text {. }
$$

We say a formula $F$ is satisfied in a model $\mathcal{M}$ if $\mathcal{M} \Vdash F$. We say a formula $F$ is valid if for all strong models $\mathcal{M}$ we have that $F$ is satisfied in $\mathcal{M}$.

THEOREM 6 (Soundness). Let CS be a constant specification. If a formula $A$ is derivable in $\mathrm{JT} 45_{\mathrm{CS}}$, then $A$ is valid.

Proof. The proof is standard. Let us only show the case for the negative introspection axiom where the strong evidence property is used.

Let $\mathcal{M}=(\mathcal{E}, \nu)$ be a strong model and assume $\mathcal{M} \Vdash \neg t: A$. From $\mathcal{M} \Vdash t: A$, by the strong evidence property, we infer that $(t, A) \notin \mathcal{E}$. Thus $(? t, \neg t: A) \in \mathcal{E}$ by the closure conditions for admissible evidence relations. Again by the strong evidence property we conclude $\mathcal{M} \Vdash ? t: \neg t: A$.

EXAMPLE 7. JT45 $\mathrm{CS}$ is indeed not sound with respect to the class of all models. Let $\mathcal{E}$ be such that $(t, P) \in \mathcal{E}$ for some term $t$ and some atomic proposition $P$. Hence we can assume $(? t, \neg t: P) \notin \mathcal{E}$. Further suppose $P \notin \nu$. Thus for the model $\mathcal{M}=(\mathcal{E}, \nu)$ we have

$$
(t, P) \in \mathcal{E} \quad \text { but } \mathcal{M} \forall t: P,
$$


which means that $\mathcal{M}$ is not strong.

We also find

$$
\mathcal{M} \Vdash \neg t: P \quad \text { but } \mathcal{M} \Vdash ? t: \neg t: P \text {, }
$$

that is $\mathcal{M}$ does not satisfy the Negative Introspection axiom.

In order to establish completeness we perform a canonical model construction.

Definition 8. Let CS be a constant specification. A set $\Phi$ of formulae is called consistent if there exists a formula $A$ that is not derivable from $\Phi$ in JT45 CS. A set $\Phi$ is called maximal consistent if it is consistent and has no consistent proper extensions.

Definition 9. Let $\Gamma$ be a maximal consistent set of formulae. The canonical model $\mathcal{M}_{\Gamma}=\left(\mathcal{E}_{\Gamma}, \nu_{\Gamma}\right)$ is given by

1. $(t, F) \in \mathcal{E}_{\Gamma}$ iff $t: F \in \Gamma$

2. $p_{i} \in \nu_{\Gamma}$ iff $p_{i} \in \Gamma$

First, we show that $\mathcal{M}_{\Gamma}$ is indeed a model, that is $\mathcal{E}_{\Gamma}$ is an admissible evidence relation.

LEMMA 10. Let $\Gamma$ be a maximal consistent set of formulae. The relation $\mathcal{E}_{\Gamma}$ is an admissible evidence relation.

Proof. We have to verify that $\mathcal{E}_{\Gamma}$ satisfies the closure conditions of an admissible evidence relation. Let us only show the case for negative introspection. Assume $(t, F) \notin \mathcal{E}_{\Gamma}$. That means, by definition, that $t: F \notin \Gamma$. Since $\Gamma$ is maximal consistent, we have $\neg t: F \in \Gamma$. Moreover, every maximal consistent set contains every axiom. In particular, $\neg t: F \rightarrow ? t: \neg t: F \in \Gamma$. Since maximal consistent sets are closed under modus ponens, we find ?t: $\neg t: F \in \Gamma$. By definition, this implies $(? t, \neg t: F) \in \mathcal{E}_{\Gamma}$.

LEMma 11 (Truth lemma). Let $\Gamma$ be a maximal consistent set of formulae. For every formula $F$ we have $\mathcal{M}_{\Gamma} \Vdash F$ iff $F \in \Gamma$.

Proof. The proof is by induction on the structure of the formula $F$.

1. $F=p_{i}$. The claim follows immediately from the definition of the satisfaction relation in the canonical model:

$$
\mathcal{M}_{\Gamma} \Vdash p_{i} \text { iff } p_{i} \in \nu_{\Gamma} \text { iff } p_{i} \in \Gamma .
$$

2. The boolean cases easily follow by the induction hypothesis.

3. $F=t: A$. Assume $t: A \in \Gamma$. Then, by definition,

$$
(t, A) \in \mathcal{E}_{\Gamma} \text {. }
$$

Since $\Gamma$ is maximal consistent, it contains the reflection axiom, that is $t: A \rightarrow A \in \Gamma$. Since maximal consistent sets are closed under modus ponens, we find $A \in \Gamma$. By the induction hypothesis, we get $\mathcal{M}_{\Gamma} \Vdash A$. Together with (1), this implies $\mathcal{M}_{\Gamma} \Vdash t: A$.

To show the reverse direction we assume $t: A \notin \Gamma$. Then, by definition, $(t, A) \notin \mathcal{E}_{\Gamma}$. Hence $\mathcal{M}_{\Gamma} \forall t: A$. 
THEOREM 12 (Completeness). Let CS be a constant specification. If a formula $A$ valid, then it is derivable in $\mathrm{JT} 45_{\mathrm{CS}}$.

Proof. Assume that the formula $F$ is not derivable in $\mathrm{JT} 45_{\mathrm{CS}}$ We show that there exists a strong counter-model for $F$. Since $F$ is not derivable, the set $\{\neg F\}$ is consistent. Thus there exists a maximal consistent set $\Gamma$ that contains $\neg F$. Then we construct the canonical model $\mathcal{M}_{\Gamma}=\left(\mathcal{E}_{\Gamma}, \nu_{\Gamma}\right)$. By the Truth lemma we find $\mathcal{M}_{\Gamma} \Vdash \neg F$. Therefore, $\mathcal{M}_{\Gamma} \Downarrow F$. It remains to show that $\mathcal{M}_{\Gamma}$ is a strong model, that is it satisfies the strong evidence property. Assume $(s, A) \in \mathcal{E}_{\Gamma}$. Hence $s: A \in \Gamma$. By the Truth lemma we conclude $\mathcal{M}_{\Gamma} \Vdash s: A$.

$\S 3$. Inductively generated models. In this section we show that it is possible to inductively generate admissible evidence relations. This allows us to introduce the class of finitely generated strong models that satisfies the conditions of Lemma 1.

We need the following auxiliary definition.

Definition 13 (Rank). The rank of a term is inductively defined by:

1. $\operatorname{rk}\left(c_{i}\right):=\operatorname{rk}\left(x_{i}\right):=0$

2. $\operatorname{rk}(s+t):=\max (\mathrm{rk}(s), \mathrm{rk}(t))+1$

3. $\operatorname{rk}(s \cdot t):=\max (\mathrm{rk}(s), \mathrm{rk}(t))+1$

4. $\mathrm{rk}(! s):=\mathrm{rk}(s)+1$

5. $\operatorname{rk}(? s):=\mathrm{rk}(s)+1$

DEFINITION 14 (Inductively generated evidence relation). An evidence base $\mathcal{B}$ is a subset of $\mathrm{Tm} \times \mathrm{Fm}$. The evidence base $\mathcal{B}$ extends a constant specification CS if

$$
c: F \in C S \quad \Longrightarrow \quad(c, F) \in \mathcal{B} \text {. }
$$

We inductively generate an evidence relation $\mathcal{E}_{\mathcal{B}}$ such that $\mathcal{B} \subseteq \mathcal{E}_{\mathcal{B}}$. By induction on the natural number $i$ we define the stages $\mathcal{E}_{\mathcal{B}}^{i}$ as follows.

1. $(s, F) \in \mathcal{E}_{\mathcal{B}}^{0}$ if $(s, F) \in \mathcal{B}$

2. $(s, F) \in \mathcal{E}_{\mathcal{B}}^{i+1}$ if $(s, F) \in \mathcal{E}_{\mathcal{B}}^{i}$

3. $(s+t, F) \in \mathcal{E}_{\mathcal{B}}^{i+1}$ if $\mathrm{rk}(s+t)=i+1$ and $(s, F) \in \mathcal{E}_{\mathcal{B}}^{i}$

4. $(s+t, F) \in \mathcal{E}_{\mathcal{B}}^{i+1}$ if $\operatorname{rk}(s+t)=i+1$ and $(t, F) \in \mathcal{E}_{\mathcal{B}}^{i}$

5. $(s \cdot t, F) \in \mathcal{E}_{\mathcal{B}}^{i+1}$ if $\mathrm{rk}(s \cdot t)=i+1$ and there exists a formula $B$ with

$$
(s, B \rightarrow F) \in \mathcal{E}_{\mathcal{B}}^{i} \text { and }(t, B) \in \mathcal{E}_{\mathcal{B}}^{i}
$$

6. $(! s, F) \in \mathcal{E}_{\mathcal{B}}^{i+1}$ if $\mathrm{rk}(! s)=i+1$ and there exists a formula $F^{\prime}$ such that $F=s: F^{\prime}$ and $\left(s, F^{\prime}\right) \in \mathcal{E}_{\mathcal{B}}^{i}$

7. $(? s, F) \in \mathcal{E}_{\mathcal{B}}^{i+1}$ if $\operatorname{rk}(? s)=i+1$ and there exists a formula $F^{\prime}$ such that $F=\neg s: F^{\prime}$ and $\left(s, F^{\prime}\right) \notin \mathcal{E}_{\mathcal{B}}^{i}$

We set $\mathcal{E}_{\mathcal{B}}:=\bigcup_{i} \mathcal{E}_{\mathcal{B}}^{i}$

REMARK 15. The generation of the evidence relation is performed by a nonmonotone inductive definition. The case that deals with negative introspection has, of course, negation built in. Thus the evidence relation that we construct will not be least fixed point but only an arbitrary fixed point. In fact, admissible evidence relations for negative introspection cannot be minimal: Kuznets [18, 
Example 3.3.43] shows very nicely how to construct two incomparable evidence relations.

Lemma 16. If $(s, F) \in \mathcal{E}_{\mathcal{B}}$ and $\mathrm{rk}(s) \leq i$, then $(s, F) \in \mathcal{E}_{\mathcal{B}}^{i}$.

Proof. First observe that

$$
k \leq l \quad \Longrightarrow \quad \mathcal{E}_{\mathcal{B}}^{k} \subseteq \mathcal{E}_{\mathcal{B}}^{l} .
$$

Assume now that $(s, F) \in \mathcal{E}_{\mathcal{B}}$ and $\operatorname{rk}(s) \leq i$. Let $j$ be the least natural number such that $(s, F) \in \mathcal{E}_{\mathcal{B}}^{j}$. Now we distinguish the different cases in the definition of $\mathcal{E}_{\mathcal{B}}^{j}$.

1. $(s, F) \in \mathcal{B}$. Thus $(s, F) \in \mathcal{E}_{\mathcal{B}}^{0}$ and $(s, F) \in \mathcal{E}_{\mathcal{B}}^{i}$ follows by (2).

2. $(s, F) \in \mathcal{E}_{\mathcal{B}}^{j-1}$. This case is not possible since $j$ is minimal.

3. In all other cases we have $\operatorname{rk}(s)=j$. Thus $j \leq i$ and hence $(s, F) \in \mathcal{E}_{\mathcal{B}}^{i}$ by $(2)$.

Lemma 17. Let $\mathcal{B}$ be a evidence base extending the constant specification $\mathrm{CS}$. Then the relation $\mathcal{E}_{\mathcal{B}}$ is an admissible evidence relation.

Proof. We have to show that $\mathcal{E}_{\mathcal{B}}$ satisfies the conditions of Definition 2. The only critical case is when $(t, A) \notin \mathcal{E}_{\mathcal{B}}$. Then $(t, A) \notin \mathcal{E}_{\mathcal{B}}^{i}$ for $i=\mathrm{rk}(t)$. Since $\mathrm{rk}(? t)=\mathrm{rk}(t)+1$, we find $(? t, \neg t: A) \in \mathcal{E}_{\mathcal{B}}^{i+1}$. Thus $(? t, \neg t: A) \in \mathcal{E}_{\mathcal{B}}$.

Definition 18 (Finitely generated model). Let CS be a finite constant specification. Let $\mathcal{B}$ be a finite evidence base extending $\mathrm{CS}$ and $\nu$ be a finite valuation. Then we call $\mathcal{M}_{\mathcal{B}}=\left(\mathcal{E}_{\mathcal{B}}, \nu\right)$ a finitely generated model.

Next we are going to show that the satisfaction relation for finitely generated models is decidable. We first need an auxiliary lemma.

Lemma 19. Let $\mathcal{B}$ be a finite evidence base. For any natural number $i$ and any term $r$, the set

$$
\left\{F \mid(r, F) \in \mathcal{E}_{\mathcal{B}}^{i} \text { and } F \text { has the form } F_{1} \rightarrow F_{2}\right\}
$$

is finite.

Proof. We show the claim by induction on $i$. The base case $i=0$ holds since $\mathcal{B}$ is finite. For the induction step let $i=j+1$. We distinguish the different cases in the definition of $\mathcal{E}_{\mathcal{B}}^{i}$ and show that each case may add only finitely many formulae of the form $F_{1} \rightarrow F_{2}$.

1. $(s, F) \in \mathcal{E}_{\mathcal{B}}^{i+1}$ because $(s, F) \in \mathcal{E}_{\mathcal{B}}^{i}$. By the induction hypothesis there are only finitely many $F=F_{1} \rightarrow F_{2}$ with $(s, F) \in \mathcal{E}_{\mathcal{B}}^{i}$.

2. $(s+t, F) \in \mathcal{E}_{\mathcal{B}}^{i+1}$ because $\mathrm{rk}(s+t)=i+1$ and $(s, F) \in \mathcal{E}_{\mathcal{B}}^{i}$. By the induction hypothesis there are only finitely many $F=F_{1} \rightarrow F_{2}$ with $(s, F) \in \mathcal{E}_{\mathcal{B}}^{i}$.

3. $(s+t, F) \in \mathcal{E}_{\mathcal{B}}^{i+1}$ because $\mathrm{rk}(s+t)=i+1$ and $(t, F) \in \mathcal{E}_{\mathcal{B}}^{i}$. Similar to the previous case.

4. $(s \cdot t, F) \in \mathcal{E}_{\mathcal{B}}^{i+1}$ because $\mathrm{rk}(s \cdot t)=i+1$ and there exists a formula $B$ with

$$
(s, B \rightarrow F) \in \mathcal{E}_{\mathcal{B}}^{i} \text { and }(t, B) \in \mathcal{E}_{\mathcal{B}}^{i} .
$$


By the induction hypothesis there are only finitely many formulae $B \rightarrow F$ with $(s, B \rightarrow F) \in \mathcal{E}_{\mathcal{B}}^{i}$. Hence there can be only finitely many $F$ with $(s \cdot t, F) \in \mathcal{E}_{\mathcal{B}}^{i+1}$.

5. $(! s, F) \in \mathcal{E}_{\mathcal{B}}^{i+1}$ because $\mathrm{rk}(! s)=i+1$ and there exists a formula $F^{\prime}$ such that $F=s: F^{\prime}$ and $\left(s, F^{\prime}\right) \in \mathcal{E}_{\mathcal{B}}^{i}$. This case cannot add formulae of the form $F_{1} \rightarrow F_{2}$ since $F$ must have the form $s: F^{\prime}$.

6. $(? s, F) \in \mathcal{E}_{\mathcal{B}}^{i+1}$ because $\mathrm{rk}(? s)=i+1$ and there exists a formula $F^{\prime}$ such that $F=\neg s: F^{\prime}$ and $\left(s, F^{\prime}\right) \notin \mathcal{E}_{\mathcal{B}}^{i}$. This case cannot add formulae of the form $F_{1} \rightarrow F_{2}$ since $F$ must have the form $\neg s: F^{\prime}$.

THEOREM 20. The satisfaction relation for finitely generated models is decidable.

Proof. Let CS be a finite constant specification. Let $\mathcal{B}$ be a finite evidence base extending it and $\nu$ be a finite valuation. First we show that for any term $s$ and any formula $F$,

$$
(s, F) \in \mathcal{E}_{\mathcal{B}} \text { is decidable. }
$$

By Lemma 16 we know $(s, F) \in \mathcal{E}_{\mathcal{B}}$ if and only if $(s, F) \in \mathcal{E}_{\mathcal{B}}^{\mathrm{rk}(s)}$. Thus it is enough to show by induction on $j$ that we can decide $(s, F) \in \mathcal{E}_{\mathcal{B}}^{j}$ for every $j$. The base case $j=0$ is decidable since $\mathcal{B}$ is finite. For the case $j=i+1$ we show that all cases in the definition of $\mathcal{E}_{\mathcal{B}}^{j}$ are decidable. The only critical case is application: $\mathrm{rk}(r \cdot t)=i+1$ and there exists a formula $B$ with $(r, B \rightarrow F) \in \mathcal{E}_{\mathcal{B}}^{i}$ and $(t, B) \in \mathcal{E}_{\mathcal{B}}^{i}$. By Lemma 19 there are only finitely many formulae of the form $B \rightarrow F$ with $(r, B \rightarrow F) \in \mathcal{E}_{\mathcal{B}}^{i}$. For all these formulae $(t, B) \in \mathcal{E}_{\mathcal{B}}^{i}$ is decidable by the induction hypothesis. Hence we have established (3).

Let $\mathcal{M}_{\mathcal{B}}=\left(\mathcal{E}_{\mathcal{B}}, \nu\right)$. Decidability of $\mathcal{M}_{\mathcal{B}} \Vdash F$ follows by induction on the structure of $F$. We distinguish the following cases:

1. The atomic case follows from the assumption that $\nu$ is finite.

2. The boolean cases follow by the induction hypothesis.

3. $F=s: F^{\prime}$. We have that $\mathcal{M}_{\mathcal{B}} \Vdash F^{\prime}$ is decidable by the induction hypothesis and $\left(s, F^{\prime}\right) \in \mathcal{E}_{\mathcal{B}}$ is decidable by $(3)$. Hence $\mathcal{M}_{\mathcal{B}} \Vdash s: F^{\prime}$ is decidable.

Now we show that it is decidable whether a finitely generated model is a strong model. Hence the class of finitely generated strong models is recursively enumerable.

LEMMA 21. Let CS be a constant specification, $\mathcal{B}$ be an evidence base extending $\mathrm{CS}$, and $\nu$ be a valuation. Consider the model $\mathcal{M}_{\mathcal{B}}=\left(\mathcal{E}_{\mathcal{B}}, \nu\right)$. If for all terms $r$ and formulae $F$ we have

$$
(r, F) \in \mathcal{B} \quad \Longrightarrow \quad \mathcal{M}_{\mathcal{B}} \Vdash F,
$$

then $\mathcal{M}_{\mathcal{B}}$ is a strong model.

Proof. By induction on $i$ we show that $(r, F) \in \mathcal{E}_{\mathcal{B}}^{i}$ implies $\mathcal{M}_{\mathcal{B}} \Vdash F$. We distinguish the following cases:

1. $(r, F) \in \mathcal{B}$. By assumption we have $\mathcal{M}_{\mathcal{B}} \Vdash F$. 
2. $(r, F) \in \mathcal{E}_{\mathcal{B}}^{i+1}$ because of $(r, F) \in \mathcal{E}_{\mathcal{B}}^{i}$. The claim follows by the induction hypothesis.

3. $r=s+t, \operatorname{rk}(s+t)=i+1$, and $(s, F) \in \mathcal{E}_{\mathcal{B}}^{i}$. By the induction hypothesis we find $\mathcal{M}_{\mathcal{B}} \Vdash F$.

4. $r=s+t, \operatorname{rk}(s+t)=i+1$, and $(t, F) \in \mathcal{E}_{\mathcal{B}}^{i}$. By the induction hypothesis we find $\mathcal{M}_{\mathcal{B}} \Vdash F$.

5. $r=s \cdot t, \mathrm{rk}(s \cdot t)=i+1,(s, B \rightarrow F) \in \mathcal{E}_{\mathcal{B}}^{i}$, and $(t, B) \in \mathcal{E}_{\mathcal{B}}^{i}$. By the induction hypothesis we find $\mathcal{M}_{\mathcal{B}} \Vdash B \rightarrow F$ and $\mathcal{M}_{\mathcal{B}} \Vdash B$. Hence $\mathcal{M}_{\mathcal{B}} \Vdash F$.

6. $r=! s, \operatorname{rk}(! s)=i+1, F=s: F^{\prime}$ and $\left(s, F^{\prime}\right) \in \mathcal{E}_{\mathcal{B}}^{i}$. By the induction hypothesis we find $\mathcal{M}_{\mathcal{B}} \Vdash F^{\prime}$. By $\left(s, F^{\prime}\right) \in \mathcal{E}_{\mathcal{B}}^{i}$, we also get $\mathcal{M}_{\mathcal{B}} \Vdash s: F^{\prime}$.

7. $r=? s, \operatorname{rk}(? s)=i+1, F=\neg s: F^{\prime}$, and $\left(s, F^{\prime}\right) \notin \mathcal{E}_{\mathcal{B}}^{i}$. We find $\left(s, F^{\prime}\right) \notin \mathcal{E}_{\mathcal{B}}$ by Lemma 16 and thus $\mathcal{M}_{\mathcal{B}} \Downarrow s: F^{\prime}$. We conclude $\mathcal{M}_{\mathcal{B}} \Vdash \neg s: F^{\prime}$.

To show that $\mathcal{M}_{\mathcal{B}}$ is a strong model, we assume $(r, F) \in \mathcal{E}_{\mathcal{B}}$. Hence we have $(r, F) \in \mathcal{E}_{\mathcal{B}}^{i}$ for some $i$ and thus, as shown above, $\mathcal{M}_{\mathcal{B}} \Vdash F$. We finally obtain $\mathcal{M}_{\mathcal{B}} \Vdash r: F$.

LEMMA 22. Let CS be a finite constant specification, $\mathcal{B}$ be a finite evidence base extending $\mathrm{CS}$, and $\nu$ be a finite valuation. Consider the model $\mathcal{M}_{\mathcal{B}}=\left(\mathcal{E}_{\mathcal{B}}, \nu\right)$. It is decidable whether $\mathcal{M}_{\mathcal{B}}$ is a strong model.

Proof. We observe that $\mathcal{M}_{\mathcal{B}}$ is a strong model if and only if

$$
\text { for all }(r, F) \in \mathcal{B} \text { we have } \mathcal{M}_{\mathcal{B}} \Vdash F \text {. }
$$

The if-direction is given by the previous lemma. For the other direction we assume that $\mathcal{M}_{\mathcal{B}}$ is a strong model and $(r, F) \in \mathcal{B}$. Hence we have $(r, F) \in \mathcal{E}_{\mathcal{B}}$ and by the strong evidence property $\mathcal{M}_{\mathcal{B}} \Vdash r: F$ which implies $\mathcal{M}_{\mathcal{B}} \Vdash F$.

Decidability of (4) follows from Theorem 20 since $\mathcal{B}$ is finite.

COROLlary 23. The class of finitely generated strong models is recursively enumerable.

§4. Decidability. It remains to show that $\mathrm{JT} 45_{\mathrm{CS}}$ is complete with respect to the class of finitely generated strong models. To achieve this, we construct a finitely generated strong submodel of the canonical model. We need the following definitions.

Definition 24. Let $\Phi$ be a finite set of formulae. We say a term $t$ occurs in $\Phi$ if there is an $F \in \Phi$ with $s: G \in \operatorname{subfml}(F)$ such that $t$ is a subterm of $s$. We let $T_{\Phi}$ be the set of all terms that occur in $\Phi$. We set:

1. $\mathrm{C}_{\Phi}(s: F):=\left\{t: G \mid t \in T_{\Phi}\right.$ and $\left.G \in \operatorname{subfml}(F)\right\}$

2. $\mathrm{M}(\Phi):=\left\{s: F \in \Phi \mid \mathrm{C}_{\Phi}(s: F) \nsubseteq \Phi\right\}$

3. $\operatorname{deg}(\Phi):=\operatorname{card}(\mathrm{M}(\Phi))$

LEMMA 25. Let $\Phi$ be a finite set of formulae. There exists a set of formulae $\operatorname{clo}(\Phi)$ such that

1. $\Phi \subseteq \operatorname{clo}(\Phi)$,

2. $\operatorname{clo}(\Phi)$ is finite,

3. clo $(\Phi)$ is closed under subformulae,

4. if $s: F \in \operatorname{clo}(\Phi)$, then $\mathrm{C}_{\mathrm{clo}(\Phi)}(s: F) \subseteq \operatorname{clo}(\Phi)$. 
Proof. Assume that we have a fixed enumeration of all formulae. If the formula $F$ is the $i$-th formula in this enumeration, then we call $i$ the index of $F$.

Depending on this enumeration we define for each set $\Pi$ of formulae a new set $\Pi^{\prime}$ as follows. Let $s: F$ be the formula with least index such that $s: F \in \mathrm{M}(\Pi)$. We set $\Pi^{\prime}:=\Pi \cup \mathrm{C}_{\Pi}(s: F)$. If $\mathrm{M}(\Pi)$ is empty, then we set $\Pi^{\prime}:=\Pi$.

First we observe

$$
T_{\Pi^{\prime}}=T_{\Pi}
$$

The inclusion $T_{\Pi} \subseteq T_{\Pi^{\prime}}$ is trivial. For the other direction we let $r$ be a term that occurs in $\Pi^{\prime}$. If $r$ occurs in $\Pi$, we are done. Otherwise $r$ must occur in $\mathrm{C}_{\Pi}(s: F)$ with $s: F \in \mathrm{M}(\Pi)$. By definition we have

$$
\mathrm{C}_{\Pi}(s: F)=\left\{t: G \mid t \in T_{\Pi} \text { and } G \in \operatorname{subfml}(F)\right\} .
$$

We distinguish two cases.

1. There exists $t: G \in \mathrm{C}_{\Pi}(s: F)$ such that $r$ is a subterm of $t$. Since $t \in T_{\Pi}$, we also have $r \in T_{\Pi}$ and we are done.

2. Otherwise there exists $t: G \in \mathrm{C}_{\Pi}(s: F)$ such that $r$ occurs in $G$. Since $G \in \operatorname{subfml}(F)$, we also have that $r$ occurs in $F$. By $s: F \in \mathrm{M}(\Pi)$, we know $s: F \in \Pi$. Hence $r$ occurs in $\Pi$ and we are done.

Thus we have established (5).

Now we take the given set $\Phi$ and define a sequence $\Phi_{0}, \Phi_{1}, \ldots$ of sets of formulae by

1. $\Phi_{0}$ is the closure of $\Phi$ under subformulae,

2. $\Phi_{m+1}:=\Phi_{m}^{\prime}$.

We prove the following for all $j$ by induction on $j$ :

1. $\Phi \subseteq \Phi_{j}$. This easily follows from $\Phi \subseteq \Phi_{0}$ and $\Phi_{i} \subseteq \Phi_{i+1}$.

2. $\Phi_{j}$ is the closed under subformulae. $\Phi_{0}$ is closed under subformulae by definition. Let $B \in \Phi_{i+1}$ and $A \in \operatorname{subfml}(B)$. If $B \in \Phi_{i}$, then we find $A \in \Phi_{i} \subseteq \Phi_{i+1}$ by the induction hypothesis. If $B \notin \Phi_{i}$, then $B \in \mathrm{C}_{\Phi_{i}}(s: F)$ for some suitable $s: F \in \Phi_{i}$ and $B$ has the from $t: G$ with $G \in \operatorname{subfml}(F)$. If $A=B$, then $A \in \mathrm{C}_{\Phi_{i}}(s: F) \subseteq \Phi_{i+1}$. If $A \neq B$, then $A \in \operatorname{subfml}(G)$. By $G \in \operatorname{subfml}(F)$ we get $A \in \operatorname{subfml}(F)$. Since $s: F \in \Phi_{i}$ and, by the induction hypothesis, $\Phi_{i}$ is closed under subformulae, we find $A \in \Phi_{i} \subseteq \Phi_{i+1}$.

3. $\Phi_{j}$ is finite. $\Phi_{0}$ is finite since there are only finitely many subformulae of the finitely many formulae in $\Phi$. To show that $\Phi_{i+1}$ is finite we first observe that $\Phi_{i}$ is finite by the induction hypothesis. The set $C_{\Phi_{i}}(s: F)$ that is added is also finite: it contains only formulae of the form $t: G$ where $t \in T_{\Phi_{i}}$ and $G \in \operatorname{subfml}(F)$. Since $T_{\Phi_{i}}$ and $\operatorname{subfml}(F)$ are finite, also the set $\mathrm{C}_{\Phi_{i}}(s: F)$ must be finite. Hence $\Phi_{i+1}$ is finite.

Note that (5) implies for all $i, j$

$$
\mathrm{C}_{\Phi_{i}}(s: F)=\mathrm{C}_{\Phi_{j}}(s: F) .
$$

Now we show that

$$
\text { if } \operatorname{deg}\left(\Phi_{i}\right) \neq 0 \text {, then } \operatorname{deg}\left(\Phi_{i+1}\right)<\operatorname{deg}\left(\Phi_{i}\right) .
$$


Let $\Phi_{i+1}=\Phi_{i} \cup \mathrm{C}_{\Phi_{i}}(s: F)$ where $s: F \in \mathrm{M}\left(\Phi_{i}\right)$. By (6) we have

$$
\mathrm{C}_{\Phi_{i+1}}(s: F)=\mathrm{C}_{\Phi_{i}}(s: F)
$$

and hence $s: F \notin \mathrm{M}\left(\Phi_{i+1}\right)$. Thus $\operatorname{deg}\left(\Phi_{i+1}\right)<\operatorname{deg}\left(\Phi_{i}\right)$ follows if we can show

$$
r: G \in \mathrm{C}_{\Phi_{i}}(s: F) \quad \Longrightarrow \quad r: G \notin \mathrm{M}\left(\Phi_{i+1}\right) .
$$

So assume $r: G \in \mathrm{C}_{\Phi_{i}}(s: F)$ and $t: H \in \mathrm{C}_{\Phi_{i+1}}(r: G)$. That is $t \in T_{\Phi_{i+1}}$ and $H \in \operatorname{subfml}(G)$. Because of (5) we have $t \in T_{\Phi_{i}}$ and because of $G \in \operatorname{subfml}(F)$ we have $H \in \operatorname{subfml}(F)$. Thus $t: H \in C_{\Phi_{i}}(s: F)$ and, therefore, $t: H \in \Phi_{i+1}$. Hence (8) is established.

Since there cannot be an infinite descending sequence of natural numbers, (7) implies that there exists $m$ such that $\operatorname{deg}\left(\Phi_{m}\right)=0$, which means

$$
\text { if } s: F \in \Phi_{m} \text {, then } C_{\Phi_{m}}(s: F) \subseteq \Phi_{m} \text {. }
$$

Finally, we set $\operatorname{clo}(\Phi)=\Phi_{m}$.

LEMMA 26. Let $\Phi$ be a finite set of formulae and $\mathcal{B}$ be an evidence base such that $(s, F) \in \mathcal{B}$ implies $s: F \in \operatorname{clo}(\Phi)$. Further, let $r$ be a term occurring in $\operatorname{clo}(\Phi)$. Then we have for all $j$ that

$$
(r, A \rightarrow B) \in \mathcal{E}_{\mathcal{B}}^{j} \quad \text { implies } \quad r:(A \rightarrow B) \in \operatorname{clo}(\Phi) .
$$

Proof. By induction on $j$. The case $j=0$ holds by assumption. Let $j=i+1$. We distinguish the following cases.

1. $(r, A \rightarrow B) \in \mathcal{E}_{\mathcal{B}}^{i}$. The claim follows by the induction hypothesis.

2. $r=s+t$ and $(s, A \rightarrow B) \in \mathcal{E}_{\mathcal{B}}^{i}$. By the induction hypothesis we find

$$
s:(A \rightarrow B) \in \operatorname{clo}(\Phi) \text {. }
$$

Since $s+t$ is a term of $\operatorname{clo}(\Phi)$, we find $s+t:(A \rightarrow B) \in \operatorname{clo}(\Phi)$ by the conditions on $\operatorname{clo}(\Phi)$.

3. $r=s+t$ and $(t, A \rightarrow B) \in \mathcal{E}_{\mathcal{B}}^{i}$. Similar to the previous case.

4. $r=s \cdot t,(s, C \rightarrow(A \rightarrow B)) \in \mathcal{E}_{\mathcal{B}}^{i}$, and $(t, C) \in \mathcal{E}_{\mathcal{B}}^{i}$. By the induction hypothesis we know $s:(C \rightarrow(A \rightarrow B)) \in \operatorname{clo}(\Phi)$. Now we observe that

$$
r:(A \rightarrow B) \in \mathrm{C}_{\mathrm{clo}(\Phi)}(s:(C \rightarrow(A \rightarrow B)))
$$

and conclude $r:(A \rightarrow B) \in \operatorname{clo}(\Phi)$.

5. $r=! s$. This case is not possible since we consider only implications.

6. $r=? s$. This case is not possible since we consider only implications.

Definition 27. Let $\mathcal{M}=(\mathcal{E}, \nu)$ be a model and $\Phi$ a finite set of formulae that contains CS. The $\Phi$-generated submodel $\mathcal{M} \uparrow \Phi$ of $\mathcal{M}$ is defined by $(\mathcal{E}\lceil\Phi, \nu \uparrow \Phi)$ where

1. $\mathcal{E}\left\lceil\Phi\right.$ is the evidence relation generated from the base $\mathcal{B}_{\Phi}$ given by

$$
(t, F) \in \mathcal{B}_{\Phi} \text { iff } t: F \in \operatorname{clo}(\Phi) \text { and }(t, F) \in \mathcal{E},
$$

2. $\nu \uparrow \Phi$ is given by

$$
p_{i} \in \nu \uparrow \Phi \text { iff } p_{i} \in \operatorname{clo}(\Phi) \text { and } p_{i} \in \nu .
$$


REMARK 28. Since we require that $\Phi$ contains CS, the above definition guarantees that $\mathcal{B}_{\Phi}$ extends CS. Thus by Lemma 17 we know that $\mathcal{E} \uparrow \Phi$ is an admissible evidence relation and hence $\mathcal{M} \uparrow \Phi$ is indeed a model.

Moreover, by Lemma 25 we find that $\operatorname{clo}(\Phi)$ is finite. Thus $\mathcal{M} \uparrow \Phi$ is a finitely generated model.

LEMMA 29. Let $\mathcal{E}$ be an admissible evidence relation, $\Phi$ a finite set of formulae that contains $\mathrm{CS}$, and $\mathcal{E}\left\lceil\Phi\right.$ be the evidence relation generated from $\mathcal{B}_{\Phi}$. We have for all $r: F \in \operatorname{clo}(\Phi)$ that

$$
(r, F) \in \mathcal{E} \text { if and only if }(r, F) \in \mathcal{E} \uparrow \Phi .
$$

Proof. From left to right. Assume $r: F \in \operatorname{clo}(\Phi)$ and $(r, F) \in \mathcal{E}$. We have $(r, F) \in \mathcal{B}_{\Phi}$ by definition and hence $(r, F) \in \mathcal{E}\lceil\Phi$.

From right to left. We show that $(r, F) \in \mathcal{E}_{\mathcal{B}_{\Phi}}^{j}$ implies $(r, F) \in \mathcal{E}$ by induction on $j$. The case $j=0$ holds by the definition of $\mathcal{B}_{\Phi}$. Let $j=i+1$. We distinguish the following cases.

1. $(r, F) \in \mathcal{E}_{\mathcal{B}_{\Phi}}^{i}$. The claim follows by the induction hypothesis.

2. $r=s+t$ and $(s, F) \in \mathcal{E}_{\mathcal{B}_{\Phi}}^{i}$. By the closure conditions on $\operatorname{clo}(\Phi)$ and the induction hypothesis we find $(s, F) \in \mathcal{E}$. Thus $(s+t, F) \in \mathcal{E}$ follows by the closure conditions of $\mathcal{E}$.

3. $r=s+t$ and $(t, F) \in \mathcal{E}_{\mathcal{B}_{\Phi}}^{i}$. Similar to the previous case.

4. $r=s \cdot t,(s, A \rightarrow F) \in \mathcal{E}_{\mathcal{B}}^{i}$, and $(t, A) \in \mathcal{E}_{\mathcal{B}}^{i}$. By Lemma 26 we find

$$
s:(A \rightarrow F) \in \operatorname{clo}(\Phi) \text {. }
$$

Hence by the induction hypothesis we get $(s, A \rightarrow F) \in \mathcal{E}$. Moreover, by the closure conditions on $\operatorname{clo}(\Phi),(9)$ and $r: F \in \operatorname{clo}(\Phi)$ together imply $t: A \in \operatorname{clo}(\Phi)$. Thus by the induction hypothesis we infer $(t, A) \in \mathcal{E}$. Finally by the closure conditions on $\mathcal{E}$ we conclude $(s \cdot t, F) \in \mathcal{E}$.

5. $r=! s, F=s: F^{\prime}$, and $\left(s, F^{\prime}\right) \in \mathcal{E}_{\mathcal{B}_{\Phi}}^{i}$. By the closure conditions on clo $(\Phi)$ and the induction hypothesis we find $\left(s, F^{\prime}\right) \in \mathcal{E}$. Thus $\left(! s, s: F^{\prime}\right) \in \mathcal{E}$ follows by the closure conditions of $\mathcal{E}$.

6. $r=? s, F=\neg s: F^{\prime},\left(s, F^{\prime}\right) \notin \mathcal{E}_{\mathcal{B}_{\Phi}}^{i}$, and $\mathrm{rk}(r)=i+1$. By Lemma 16 we find $\left(s, F^{\prime}\right) \notin \mathcal{E}_{\mathcal{B}_{\Phi}}$. By the closure conditions on clo $(\Phi)$ we get $s: F^{\prime} \in \operatorname{clo}(\Phi)$. Hence by the direction from left to right we obtain $\left(s, F^{\prime}\right) \notin \mathcal{E}$. Thus $\left(? s, \neg s: F^{\prime}\right) \in \mathcal{E}$ follows by the closure conditions on $\mathcal{E}$.

Lemma 30. Let $\mathcal{M}=(\mathcal{E}, \nu)$ be a model and $\Phi$ be a finite set of formulae that contains CS. Further, let $\mathcal{M} \uparrow \Phi$ be the $\Phi$-generated submodel of $\mathcal{M}$. Then for all formulae $F \in \operatorname{clo}(\Phi)$ we have

$$
\mathcal{M} \uparrow \Phi \Vdash F \text { if and only if } \mathcal{M} \Vdash F \text {. }
$$

Proof. Proof by induction on the structure of $F$.

1. $F=p_{i}$. We have $\mathcal{M} \uparrow \Phi \Vdash p_{i}$ iff $p_{i} \in \nu \uparrow \Phi$ and $p_{i} \in \operatorname{clo}(\Phi)$. Since $p_{i} \in \operatorname{clo}(\Phi)$ by assumption, this is equivalent to $p_{i} \in \nu$ which is $\mathcal{M} \Vdash p_{i}$.

2. The boolean cases follow immediately by the induction hypothesis. 
3. $F=s: F^{\prime}$. We have $\mathcal{M}\left\lceil\Phi \Vdash s: F^{\prime}\right.$ if and only if

$$
\text { (a) }\left(s, F^{\prime}\right) \in \mathcal{E} \uparrow \Phi \quad \text { and } \quad\left(\text { b) } \mathcal{M} \uparrow \Phi \Vdash F^{\prime}\right. \text {. }
$$

Since $s: F^{\prime} \in \operatorname{clo}(\Phi)$, we find by Lemma 29 that (a) is equivalent to (a') $\left(s, F^{\prime}\right) \in \mathcal{E}$. Moreover (b) is equivalent to (b') $\mathcal{M} \Vdash F^{\prime}$ by the induction hypothesis. Finally (a') and (b') together are equivalent to $\mathcal{M} \Vdash s: F^{\prime}$.

LEMma 31. Let $\mathcal{M}=(\mathcal{E}, \nu)$ be a model, $\Phi$ a finite set of formulae that contains $\mathrm{CS}$, and $\mathcal{M}\lceil\Phi$ the $\Phi$-generated submodel of $\mathcal{M}$. If $\mathcal{M}$ is a strong model, then $\mathcal{M} \uparrow \Phi$ is a strong model, too.

Proof. By Lemma 21 it is enough to show

$$
(t, F) \in \mathcal{B}_{\Phi} \quad \Longrightarrow \quad \mathcal{M} \uparrow \Phi \Vdash F \text {. }
$$

So assume $(t, F) \in \mathcal{B}_{\Phi}$. That is (a) $t: F \in \operatorname{clo}(\Phi)$ and $(\mathrm{b})(t, F) \in \mathcal{E}$. Since $\operatorname{clo}(\Phi)$ is closed under subformulae we have (a') $F \in \operatorname{clo}(\Phi)$. Since $\mathcal{M}$ is a strong model (b) implies $\mathcal{M} \Vdash t: F$ from which we get (b') $\mathcal{M} \Vdash F$. From (a') and (b') we conclude by Lemma 30 that $\mathcal{M} \uparrow \Phi \Vdash F$. Hence (10) is established.

THEOREM 32. Let CS be a finite constant specification. Let $F$ be a formula that is not derivable in $\mathrm{JT}_{4} 5_{\mathrm{CS}}$. Then there exists a finitely generated strong model $\mathcal{M}_{\mathcal{B}}$ with $\mathcal{M}_{\mathcal{B}} \| F$.

Proof. By Theorem 12 we find a strong model $\mathcal{M}$ with $\mathcal{M} \Downarrow F$. Let $\Phi$ be the union of $\{F\}$ and CS. Since $\Phi$ is finite, we know that $\mathcal{M}\lceil\Phi=(\mathcal{E}\lceil\Phi, \nu \uparrow \Phi)$ is a finitely generated model. By Lemma 31 we know that $\mathcal{M} \uparrow \Phi$ is a strong model. Moreover, by Lemma 30 we find $\mathcal{M}\lceil\Phi \mid \forall F$. Thus the claim follows by setting $\mathcal{M}_{\mathcal{B}}:=\mathcal{M}\lceil\Phi$.

COROLlaRY 33. JT45 CS is decidable for finite constant specifications CS.

Proof. Let $\mathcal{C}$ be the class of finitely generated strong models. By Theorem 6 know that $\mathrm{JT} 45_{\mathrm{CS}}$ is sound with respect to $\mathcal{C}$ and Theorem 32 gives us completeness of $\mathrm{JT} 45_{\mathrm{CS}}$ with respect to $\mathcal{C}$. The class $\mathcal{C}$ is recursively enumerable by Corollary 23. Finally, by Theorem 20, the binary relation $\mathcal{M} \Vdash F$ between formulae and models from $\mathcal{C}$ is decidable. Thus we have established the assumptions of Lemma 1 and conclude that JT45 CS is decidable.

§5. Discussion. We have established decidability of $\mathrm{JT} 45_{\mathrm{CS}}$ for finite constant specifications CS. Our method also applies to the sublogics $\mathrm{JT} 5_{\mathrm{CS}}, \mathrm{J45}$ CS, and $\mathrm{J} 5_{\mathrm{CS}}$ that are given as follows.

JT5 $5_{C S}$ is obtained from $\mathrm{JT}_{4} 5_{\mathrm{CS}}$ by dropping the Positive Introspection axiom. On the semantic side, we drop the clause for the !-operator in the definition of an admissible evidence relation. Decidability of JT5CS is established essentially in the same way as decidability of $\mathrm{JT} 45_{\mathrm{CS}}$, simply delete all cases dealing with the !-operator.

J45CS is obtained from $\mathrm{JT}_{4} 5_{\mathrm{CS}}$ by dropping the Reflection axiom. On the semantic side, we adapt the satisfaction relation such that of $\mathcal{M} \Vdash t: A$ if and only if $(t, A) \in \mathcal{E}$ (i.e. we drop the additional condition $\mathcal{M} \Vdash A$ ). Using this 
definition of satisfaction in a model, the strong evidence property trivializes. Again, we can show decidability as above. However, we do not need to take care of the strong evidence property. In particular, we do not need Lemma 21 and Lemma 31.

$\mathrm{J} 5_{\mathrm{CS}}$ is obtained from $\mathrm{J45} \mathrm{CS}$ by dropping the Positive Introspection axiom. Again, we drop all cases dealing with the !-operator in the treatment of $\mathrm{J45}$ CS and easily obtain decidability of $\mathrm{J} 5 \mathrm{CS}$.

THEOREM 34. The logics $\mathrm{J} 5_{\mathrm{CS}}$, $\mathrm{J} 45_{\mathrm{CS}}$, $\mathrm{JT} 5_{\mathrm{CS}}$, and $\mathrm{JT} 45_{\mathrm{CS}}$ are decidable for finite constant specifications CS.

Often, decidability for a justification logic is not established for finite constant specifications but for schematic constant specifications that are defined as follows.

Definition 35. A constant specification is called schematic if for every constant $c$ the set $\{F \mid c: F\}$ consists of several (possibly zero) axiom schemes.

It is open whether our approach can be adapted to deal with schematic constant specifications. Essentially, there are two problems when the constant specification CS is schematic.

1. We cannot decide whether $\mathcal{M}_{\mathcal{B}}$ is a strong model, see Lemma 22. Our proof requires that CS is a finite set since we check for each element of CS whether is satisfies the strong evidence property.

2. We cannot decide the satisfaction relation for finitely generated models, see Theorem 20. The proof of that theorem relies on the fact that a given evidence term can justify only finitely many implications (Lemma 19), which, of course, is not the case for schematic constant specifications. The usual 'trick' of working with schemes in the construction of the evidence relation does not work either: even if there are only finitely many schemes $A \rightarrow F$, we would have to check whether there is a most general unifier of $A$ and infinitely many schemes $B$.

§6. Conclusion. Justification logics are modal logics that include justifications for the agent's knowledge. So far, there were no decidability results available for justification logics with negative introspection. To address this issue, we have developed a novel model construction for such logics. In particular we have shown how to inductively build a kind of M-model for negative introspection.

Assuming a finite constant specification, we have defined the class of finitely generated strong models and established that this class is recursively enumerable and that the satisfaction relation for its models is decidable. Using a new submodel construction, we have also been able to show that JT45 CS is complete with respect to that class. Hence $J \mathrm{~T} 45_{\mathrm{CS}}$ is decidable. Our technique also works for $\mathrm{J} 5 \mathrm{CS}, \mathrm{J} 45_{\mathrm{CS}}$, and $\mathrm{JT} 5_{\mathrm{CS}}$.

The main future task in this line of research is to solve the decision problem for justification logics with negative introspection and schematic constant specifications. This will also include the treatment of logics with the D-axiom for which we have to adapt the methods to deal with so-called F-models [10]. Moreover, we believe that the tools we have developed in this paper will also allow 
us to decide more complex logics like an extension of justifications for common knowledge [8] with negative introspection.

\section{REFERENCES}

[1] Sergei N. Artemov, Operational modal logic, Technical Report MSI 95-29, Cornell University, December 1995.

[2] - Explicit provability and constructive semantics, The Bulletin of Symbolic Logic, vol. 7 (2001), no. 1, pp. 1-36.

[3] Sergei [N.] Artemov, The logic of justification, The Review of Symbolic Logic, vol. 1 (2008), no. 4, pp. 477-513.

[4] S[ergei N.] Artemov, E. [L.] Kazakov, and D. Shapiro, Logic of knowledge with justifications, Technical Report CFIS 99-12, Cornell University, 1999.

[5] Sergei [N.] Artemov and Roman Kuznets, Logical omniscience as a computational complexity problem, Theoretical Aspects of Rationality and Knowledge, proceedings of the twelfth conference (TARK 2009) (Stanford University, California) (Aviad Heifetz, editor), ACM, July 6-8, 2009, pp. 14-23.

[6] Sergei [N.] Artemov and Elena Nogina, Logic of knowledge with justifications from the provability perspective, Technical Report TR-2004011, CUNY Ph.D. Program in Computer Science, August 2004.

[7] Samuel Bucheli, Roman Kuznets, Bryan Renne, Joshua Sack, and Thomas STUDER, Justified belief change, LogKCA-10, proceedings of the second ILCLI international workshop on logic and philosophy of knowledge, communication and action (Xabier Arrazola and María Ponte, editors), University of the Basque Country Press, 2010, pp. 135155.

[8] Samuel Bucheli, Roman Kuznets, and Thomas Studer, Justifications for common knowledge, Journal of Applied Non-Classical Logics, vol. 21 (2011), no. 1, pp. 35-60.

[9] - Partial realization in dynamic justification logic, Logic, Language, Information and Computation, 18th international Workshop, WoLLIC 2011, Philadelphia, PA, USA, May 18-20, 2011, proceedings (Lev D. Beklemishev and Ruy de Queiroz, editors), Lecture Notes in Artificial Intelligence, vol. 6642, Springer, 2011, pp. 35-51.

[10] Melvin Fitting, The logic of proofs, semantically, Annals of Pure and Applied Logic, vol. 132 (2005), no. 1, pp. 1-25.

[11] Gerhard JäGer and Thomas Studer, Extending the system $\mathrm{T}_{0}$ of explicit mathematics: the limit and Mahlo axioms, Annals of Pure and Applied Logic, vol. 114 (2002), no. $1-3$, p. $79-101$.

[12] Vladimir N. KRupski, Operational logic of proofs with functionality condition on proof predicate, Logical Foundations of Computer Science, 4th international symposium, LFCS'97, Yaroslavl, Russia, July 6-12, 1997, proceedings (Sergei Adian and Anil Nerode, editors), Lecture Notes in Computer Science, vol. 1234, Springer, 1997, pp. 167-177.

[13] - The single-conclusion proof logic and inference rules specification, Annals of Pure and Applied Logic, vol. 113 (2001), no. 1-3, pp. 181-206.

[14] - Reference constructions in the single-conclusion proof logic, Journal of Logic and Computation, vol. 16 (2006), no. 5, pp. 645-661.

[15] — Referential logic of proofs, Theoretical Computer Science, vol. 357 (2006), no. $1-3$, pp. $143-166$

[16] Roman Kuznets, On the complexity of explicit modal logics, Computer Science Logic, 14th international workshop, CSL 2000, annual conference of the EACSL, Fischbachau, Germany, August 21-26, 2000, proceedings (Peter G. Clote and Helmut Schwichtenberg, editors), Lecture Notes in Computer Science, vol. 1862, Springer, 2000, pp. 371-383.

[17] - On decidability of the logic of proofs with arbitrary constant specifications, 2004 annual meeting of the Association for Symbolic Logic, Carnegie Mellon University, Pittsburgh, PA, May 19-23, 2004, Bulletin of Symbolic Logic, vol. 11(1), Association for Symbolic Logic, March 2005, Abstract, p. 111. 
[18] — Complexity issues in justification logic, Ph.D. thesis, CUNY Graduate Center, May 2008.

[19] Roman Kuznets and Thomas Studer, Justifications, ontology, and conservativity, Advances in modal logic, volume 9 (Thomas Bolander, Torben Braüner, Silvio Ghilardi, and Lawrence Moss, editors), College Publications, 2012, pp. 437-458.

[20] Alexey Mkrtychev, Models for the logic of proofs, Logical Foundations of Computer Science, 4th international symposium, LFCS'97, Yaroslavl, Russia, July 6-12, 1997, proceedings (Sergei Adian and Anil Nerode, editors), Lecture Notes in Computer Science, vol. 1234, Springer, 1997, pp. 266-275.

[21] ERIC Pacuit, A note on some explicit modal logics, Proceedings of the 5th Panhellenic Logic Symposium (Athens, Greece), University of Athens, July 25-28, 2005, pp. 117125.

[22] Wayne Richter and Peter Aczel, Inductive definitions and reflecting properties of admissible ordinals, Generalized recursion theory - proceedings of the 1972 oslo symposium (J.E. Fenstad and P.G. Hinman, editors), Studies in Logic and the Foundations of Mathematics, vol. 79, Elsevier, 1974, pp. 301-381.

[23] Natalia [M.] RubTsova, On realization of S5-modality by evidence terms, Journal of Logic and Computation, vol. 16 (2006), no. 5, pp. 671-684.

[24] Tatiana [L.] Sidon, Provability logic with operations on proofs, Logical Foundations of Computer Science, 4th international symposium, LFCS'97, Yaroslavl, Russia, July 6-12, 1997, proceedings (Sergei Adian and Anil Nerode, editors), Lecture Notes in Computer Science, vol. 1234, Springer, 1997, pp. 342-353.

[25] Tatiana Yavorskaya (Sidon), Logic of proofs and provability, Annals of Pure and Applied Logic, vol. 113 (2001), no. 1-3, pp. 345-372.

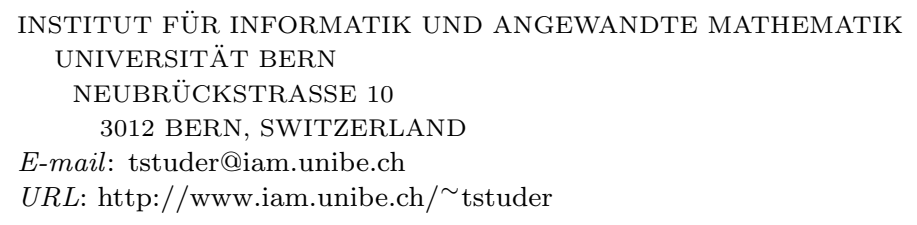

